\title{
Variations
}

Variations Revue internationale de théorie critique

\section{Meurtre, héritage traumatique, transmission}

\section{David Benhaim}

\section{OpenEdition}

\section{Journals}

Édition électronique

URL : http://journals.openedition.org/variations/244

DOI : 10.4000/variations. 244

ISSN : 1968-3960

\section{Éditeur}

Les amis de Variations

\section{Édition imprimée}

Date de publication : 21 décembre 2008

\section{Référence électronique}

David Benhaim, « Meurtre, héritage traumatique, transmission », Variations [En ligne], 12 | 2008, mis en ligne le 01 janvier 2012, consulté le 20 avril 2019. URL : http://journals.openedition.org/variations/244 ; DOI : 10.4000/variations.244

Ce document a été généré automatiquement le 20 avril 2019.

Les ami•e•s de Variations 


\title{
Meurtre, héritage traumatique, transmission
}

\author{
David Benhaim
}

\section{NOTE DE L'ÉDITEUR}

Première publication sur www.theoriecritique.com, « Wärmestrom, le courant chaud en sciences humaines ", Hiver 2008/2009, pp. 66-74

\section{Le meurtre}

1 Les exterminations et les génocides qui ont marqué le $\mathrm{XX}^{\mathrm{e}}$ siècle ne pouvaient laisser les analystes indifférents. Il était impérieux de penser ces événements et de comprendre quelque chose à l'histoire qu'ils étaient en train de vivre. C'est Nathalie Zaltzman qui a certainement exprimé le mieux cette position lorsqu'elle affirme la nécessité pour les analystes à intégrer dans le corpus analytique les conséquences pour chacun, dans son for intérieur, dans et hors sa pratique, ces conséquences d'un événement majeur collectif et individuel, postérieur à la métapsychologie freudienne de Psychologie des foules et analyse du moi : celui de l'écroulement advenu de la civilisation occidentale dans sa fonction de rempart de l'individu contre le règne du meurtre ${ }^{1}$.

2 Qu'est-ce qui s'est écroulé ? L'évidence que l'homme possédait à sa naissance d'être un homme pour l'homme. Un pacte existait, écrit-elle, non sans illusion, mais il existait; il garantissait l'assise identificatoire commune, certaine ${ }^{2}$. C'est cette certitude qui s'est écroulée et cet écroulement fait maintenant partie de l'héritage de la réalité humaine.

3 Pour Freud, l'origine de la Kultur est traumatique: Totem et tabou, texte paradigmatique sur le meurtre, élaborera l'idée d'un meurtre à l'origine de l'humanité, un crime commis en commun. Ce meurtre est un acte fondateur qui déclenche un mouvement irréversible à partir duquel va s'opérer le passage de la nature à la culture, avènement de la civilisation. Vingt-cinq ans plus tard, L'Homme Moïse et la religion monothéiste évoquera l'origine 
également traumatique du monothéisme juif: la fin brutale de Moïse à la suite du soulèvement du peuple. De L'interprétation du rêve à L'Homme Moïse, Freud élabore l'idée de meurtre dans sa double dimension de désir de mort et de meurtre accompli. À la fin du chapitre sept de L'interprétation du rêve, évoquant le mot de Platon, à savoir que le vertueux se contente de rêver de ce que le méchant fait dans la vie, Freud revient sur les désirs de mort en insistant particulièrement sur la nécessité de maintenir séparés les deux registres de la réalité psychique et de la réalité matérielle.

4 À la veille de la deuxième guerre mondiale, il écrira dans L'Homme Moïse et la religion monothéiste: Nous vivons en un temps particulièrement curieux. Nous découvrons avec surprise que le progrès a conclu un pacte avec la barbarie ${ }^{3}$. Une lecture après-coup des Considérations actuelles sur la guerre et sur la mort fait apparaître les analyses freudiennes sur la guerre comme une illustration et un développement de cette affirmation. C'est aussi ce qu'Adorno retiendra de sa lecture de Malaise et de Psychologie des foules et analyse du moi lorsqu'il écrira dans Éduquer après Auschwitz:

(...) la civilisation engendre l'anticivilisation et ne cesse de la renforcer [...] Si la barbarie s'inscrit dans le principe même de la civilisation, il peut sembler désespéré de vouloir s'y opposer ${ }^{4}$.

5 Dans les Considérations, Freud déclare que nous descendons d'une lignée infiniment longue de meurtriers qui avaient dans le sang le plaisir au meurtre, comme peut-être nous-mêmes encore ${ }^{5}$. L'homme originaire survit en nous, dans notre inconscient, inchangé. Ainsi comparant l'homme des origines aux autres animaux, il écrit :

«[...] plus cruel et plus mauvais que d'autres animaux. Il pratiquait le meurtre volontiers et comme allant de soi. L'instinct qui retient d'autres bêtes de tuer et de dévorer des êtres de la même espèce, rien ne nous permet de le lui attribuer ${ }^{6}$."

6 Cependant ce texte représente un renversement dans la façon de considérer le meurtre. Si jusque-là Freud distinguait nettement la réalité matérielle de la réalité psychique, si le meurtre se déroulait sur une scène intérieure, la scène psychique - scène du rêve ou du fantasme -, sa réflexion sur la guerre va complètement bouleverser cette manière de voir. La guerre efface cet écart, réalité psychique et réalité matérielle viennent à se confondre, le meurtre se joue alors sur la scène extérieure. La guerre, en nous dépouillant des acquisitions de la civilisation, fruit du long processus d'hominisation, met à nu cet homme originaire qui est en nous. Avec elle, nous assistons à l'écroulement de l'interdit du meurtre. Cet interdit, écrit Nathalie Zaltzman,

est la condition de la vie des hommes entre eux. En tant que psychanalystes nous tenons cet interdit, cette loi, pour une structure psychique de base. Mais alors, quelle représentation, quel contenu, quel statut occupe ou acquiert un homme pour un autre homme lorsqu'il est mis au ban, situé comme exclu de cet interdit général, désigné comme tuable, sans que ce soit un meurtre, tuable hors transgression, hors sanction, impunément tuable ${ }^{7}$ ?

En écho à cette interrogation, Janine Altounian écrit :

(...) les massacres contemporains par leurs semblables (aux antipodes des frères coalisés) de pères et de mères par millions ne peuvent qu'endommager irrémédiablement l'identité sexuelle et culturelle de leurs enfants : à côté de la dignité des pères mis à mort se transmet aussi, à leurs fils et filles, la honte de leur destitution impunément effectuée, l'empreinte de leurs corps publiquement violés, car, avant le meurtre, le corps est toujours violenté. C'est justement en tant que différents qu'ils furent exterminés et la seule différence qui, pour eux, demeura signifiante ne fut pas celle d'être resté homme ou femme, mais celle d'en revenir mort ou vivant ${ }^{8}$. 


\section{Trauma et héritage traumatique}

De tels meurtres, une telle cruauté sanglante, une telle désolation nous laissent sans voix et sans parole. La capacité de penser ne peut qu'être sidérée. Nous sommes envahis à la fois par une douleur et une rage sans nom. Je pense à la terreur sans nom dont parle Bion pour désigner l'éprouvé du nourrisson lorsque la mère, parce qu'elle n'a pas ce qu'il appelle la capacité de contenance et de rêverie, est incapable de métaboliser les contenus qu'il projette en elle et qu'il s'identifie alors à un objet incapable de comprendre. Je pense aussi à cette angoisse atavique dont parle Primo Levi, celle dont on entend l'écho au deuxième verset de la genèse : l'angoisse inscrite en chacun de nous du " tohu-bohu ", de l'univers désert et vide, écrasé sous l'esprit de Dieu, mais dont l'esprit de l'homme est absent : ou pas encore né ou déjà éteint. Terreur sans nom, angoisse atavique sont des manières de dire quelque chose du trauma, un trauma qui plus que tout autre opère une telle rupture qu'il expose les victimes à un débordement incontrôlable de tout le système de défenses disponibles, qui ne se limitent pas à la liste des défenses par mentalisation'. Mais le modèle de traumatisme que la psychanalyse nous a légué, peut-il nous aider à penser cet événement ? Cette question fait écho à cette mise en garde redoutable que faisait Primo Levi aux psychanalystes lorsqu'il écrivait dans Les naufragés et les rescapés :

\footnotetext{
Je ne crois pas, que les psychanalystes (qui se sont jetés sur nos problèmes embrouillés avec une avidité professionnelle) soient compétents pour expliquer cette impulsion [il fait allusion à l'écriture témoignage que constitue la littérature concentrationnaire]. Leur savoir a été construit et mis à l'épreuve "au-dehors", dans le monde que, pour simplifier, nous appelions "civilisé»: il en décalque la phénoménologie et tente de l'expliquer; il en étudie les déviations et s'efforce de les guérir. Leurs interprétations, même celles de ceux qui, tel Bruno Bettelheim, ont traversé l'épreuve du Lager, me paraissent approximatives et simplifiées, un peu comme si quelqu'un voulait appliquer les théories de la géométrie plane à la résolution des triangles sphériques. Les mécanismes mentaux des Häftlinge étaient différents des nôtres.

(...) Les ulcères gastriques et les maladies mentales guérissaient (ou devenaient asymptomatiques), mais tous souffraient d'un trouble continuel qui empoisonnait le sommeil et qui ne porte pas de nom. Le définir « névrose » serait réducteur et ridicule ${ }^{10}$.
}

\section{Qu'est-ce que la pensée psychanalytique entend par traumatisme?}

9 Pour situer la notion, je dirai qu'elle oscille entre deux conceptions marquées par le désaccord, présentes depuis les origines de la psychanalyse et dont les conséquences retentissent toujours sur la psychanalyse contemporaine. Deux noms peuvent être assignés à ces conceptions : Freud et Ferenczi. À quoi tient leur désaccord ? À la place de l'événement ou des événements à l'origine du traumatisme. Freud croira au départ à la réalité des scènes de séduction, mais sera amené ensuite à dissocier la réalité événementielle de la réalité psychique. Ainsi ce qu'il aura identifié au départ comme des scènes de séduction réelle, il finira par les considérer comme des créations fantasmatiques, des mensonges de l'enfant. Il s'agit, comme il l'écrira lui-même dans Autres remarques sur les psychonévroses de défense, de la distinction entre souvenirs réels et fantasmes des analysés, relatifs à leurs années d'enfance. Dans sa lettre à Fliess du 21 septembre 1897, parmi les motifs qu'il invoque pour l'abandon de sa théorie de la séduction, il exprime sa conviction qu'il n'existe dans l'inconscient aucun "indice de réalité " 
de telle sorte qu'il est impossible de distinguer l'une de l'autre la vérité de la fiction investie d'affect ${ }^{11}$. C'est dans cette même lettre qu'il écrira: Il faut que je te confie tout de suite le grand secret qui, au cours de ces derniers mois, s'est lentement révélé. Je ne crois plus à ma "neurotica". Trente-cinq ans plus tard, en 1932, Ferenczi s'opposera à Freud, et ce, jusqu'à sa mort, pour affirmer la réalité événementielle de ces scènes et l'origine extérieure traumatique de la névrose. C'est dans son article Confusion de langues entre l'adulte et l'enfant qu'il consignera ses objections et exposera sa conception du traumatisme. En fait sa position constitue un retour à la neurotica. Quant aux conséquences sur la pensée psychanalytique, nul mieux que Balint n'a exprimé l'effet lorsqu'il écrit dans Le défaut fondamental : « Le fait historique représenté par le désaccord entre Freud et Ferenczi fit sur le monde analytique l'effet d'un traumatisme ${ }^{12}$.»

Comment alors définir le traumatisme? Alors qu'il était passé au second plan dans les préoccupations de Freud, les névroses de guerre le ramèneront au premier plan sous la forme clinique des névroses traumatiques et l'imposeront de nouveau à son attention. C'est l'Au-delà du principe de plaisir qui témoigne de cet intérêt. Il reprendra sa définition du traumatisme comme effraction et sera conduit à formuler l'hypothèse qu'un afflux excessif d'excitation met d'emblée hors jeu le principe de plaisir, contraignant l'appareil psychique à accomplir une tâche plus urgente "au-delà du principe de plaisir ", tâche qui consiste à lier les excitations de façon à permettre ultérieurement leur décharge ${ }^{13}$.

Dans Inhibition, symptôme et angoisse Freud envisage le traumatisme dans ce qui en est sa condition déterminante fondamentale : la perte de l'objet. Nous ne sommes plus alors dans le registre de la séduction. C'est l'état de détresse ou le désaide du nourrisson qui va lui permettre d'aborder la question du traumatisme. Qu'est-ce que l'état de détresse ? C'est l'état du nourrisson qui, étant donné sa prématuration, est impuissant à mettre un terme à l'accroissement de tension de ses besoins par une action spécifique et par suite est entièrement dépendant d'autrui pour leur satisfaction. Il s'agit d'un prototype de situation traumatique qui est générateur d'angoisse. L'absence éprouvée de la mère, lorsque la tension des besoins s'accroit, plonge le nourrisson dans un état de détresse qui se caractérise par un afflux d'excitations qu'il ne peut maîtriser. Sa réponse est ce que Freud appelle une angoisse automatique. Lorsque cette situation se reproduit, le moi met en action un dispositif appelé signal d'angoisse qui permet de faire face à la situation redoutée ; l'angoisse se déclenche avant que la situation ne se produise. Dans les deux cas, elle apparaît comme l'expression de l'état de détresse psychique du nourrisson face à son état de détresse biologique.

12 Cependant si cet état semble correspondre à une phase du développement, on ne peut pas éviter de le voir comme un état qui se répète dans tous les cas où l'être humain est dans l'incapacité de s'aider, de se protéger lui-même contre des forces supérieures qu'il ne saurait maitriser. Bruno Bettelheim l'exprime pertinemment lorsqu'il écrit: Le traumatisme du camp - avoir été impuissant, sans contrôle sur sa vie, anéanti $i^{1415}$

A partir de là, je poserai deux questions : d'abord qu'est-ce qu'on entend par héritage traumatique? Ensuite qu'est-ce qu'on transmet lorsqu'on transmet un héritage traumatique ?

14 Parler d'héritage implique que nous nous situions dans une perspective inter- ou transgénérationnelle. Dans la succession des générations, chaque famille transmet à ses membres l'héritage acquis, sa façon d'appréhender le monde et d'organiser son monde interne, tout en les ouvrant aussi à la dimension historique. À l'instar de l'appareil 
psychique groupal conçu par René Kaës, André Ruffiot a forgé l'idée d'un appareil psychique familial. Il "a d'abord décrit l'appareil psychique familial, fait de psyché pure, précédant l'ancrage corporel des psychismes individuels, comme perdurant à travers les générations. C'est un appareil à transmettre ${ }^{16}$. Il permet d'articuler les deux axes structuraux de la famille: l'axe horizontal constitutif de la trame synchronique qui se définit par le groupe parents/enfants et l'axe vertical, chaîne diachronique qui renvoie à la dimension générationnelle, à la succession des générations et à la transmission psychique entre elles. L'individu ne peut construire son histoire que s'il s'enracine solidement dans l'histoire familiale qui le précède et qui va nourrir ses assises narcissiques, histoire dans laquelle il va prendre sa place de sujet. Je ne puis que renvoyer ici à la notion de contrat narcissique développé par Piera Aulagnier dans La violence de l'interprétation et reprise par René Kaës. Chaque sujet vient au monde psychique, social et générationnel porteur d'une mission qui consiste à assurer la continuité de l'ensemble auquel il appartient. Cet ensemble investira narcissiquement le sujet. Ce dernier devra reprendre et transmettre les énoncés fondamentaux du mythe fondateur de l'ensemble même si ceux-ci entrent en contradiction avec ce que lui-même perçoit.

Je distinguerai un double héritage: l'héritage intergénérationnel et l'héritage transgénérationnel. Le premier est constitué d'éléments élaborés: fantasmes, identifications qui organisent une histoire familiale, un récit mythique dans lequel chaque sujet peut puiser les éléments nécessaires à la constitution de son roman familial individuel ${ }^{17}$. Ce qui se transmet (...) c'est aussi ce qui soutient et assure, écrit René Kaës, les continuités narcissiques, le maintien des liens intersubjectifs, la conservation des formes et des processus de conservation et de complexification de la vie: idéaux, mécanismes de défense, identifications, pensées de certitudes, doutes ${ }^{18}$. Quant au deuxième, il est constitué d'éléments bruts, non élaborés. Il consiste en vécus traumatiques, en deuils non faits. L'objet transmis n'a pas été élaboré par l'ancêtre ; il est ainsi transmis à l'état brut, sans transformation ; celui qui le reçoit se retrouve avec un objet étrange et étranger, mutique, difficilement transformable sinon impossible à transformer tel un objet inanimé que le sujet ne peut s'approprier. Dans la continuité de la pensée de Freud, René Kaës écrit :

" (...) ce qui se transmet, ce n'est pas seulement le positif. Nous savons mieux aujourd'hui que ce qui se transmet, dans la transsubjectivité des générations, des couples et des groupes, c'est ce qui fait défaut, ce qui manque, ce qui n'a pas reçu d'inscription, ce dont l'inscription a été empêchée, ce qui a été nié, refoulé ou forclos : au prix d'un meurtre silencieux, au prix d'un blanc, d'un trou, d'une éclipse de l'être ${ }^{19}$.»

\section{Transmission}

Dans les dernières pages de Totem et tabou, Freud formule deux présuppositions qui étaient restées implicites dans ses analyses antérieures. La première, qui a servi de fondement à toute sa construction et qu'il qualifie d'audacieuse, est celle d'une psyché de masse dans laquelle les processus psychiques s'accomplissent comme dans la vie psychique d'un individu. Cette hypothèse lui est indispensable pour expliquer la perpétuation sinon l'efficacité du sentiment de culpabilité au cours des siècles et surtout pendant des générations qui ne connurent pas le meurtre du père originaire et n'en pouvaient rien savoir. Quelles que soient les réserves que l'on puisse avoir à l'égard d'une telle présupposition, on est obligé de reconnaître qu'elle rend possible de penser « une continuité dans la vie affective des hommes qui permette de faire abstraction des interruptions des actes psychiques entraînés par le fait que les individus passent $t^{20}$. » 
Sans cette continuité il n'existerait ni progrès ni évolution, chaque nouvelle génération aurait à recommencer, à acquérir tout ce que les générations antérieures ont acquis. Freud pose alors deux questions. La première concerne la part attribuée à la continuité psychique dans la suite des générations ; la seconde touche aux voies et aux moyens dont se sert une génération pour transmettre ses états psychiques à la suivante. Sa réponse précise deux choses : la tradition telle qu'ordinairement comprise, c'est-à-dire comme transmission directe, est écartée car elle n'apporte pas de réponse satisfaisante. Il repensera cette notion de tradition dans la troisième partie de L'Homme Moïse en rapport avec le monothéisme juif. C'est plutôt l'hérédité des dispositions psychiques qui assure cette continuité; cependant pour être efficientes, elles ont besoin de recevoir une stimulation de la vie individuelle. Freud invoque les paroles de Goethe dans le Faust:

Ce que tu as hérité de tes pères, /Acquiers-le afin de le posséder,

Mais il omet le vers suivant, Ce dont on n'use pas est un lourd fardeau, qui est une expression de l'héritage traumatique. Dans La survivance, Janine Altounian le commente ainsi :

«Ce matériau brut, sédimentation du trauma parental dans le psychisme de l'enfant doit être utilisé, mis en activité, exploité par lui tel un gisement explosible à désamorcer. Il doit se transformer en matière à représentation, à nomination, pour que soit enfin levée l'hypothèse de son pesant fardeau. Si cette tâche lancinante alimente la plupart des écrits des enfants de survivants aux génocides, persécutions et multiples violences de notre siècle, il est à noter qu'elle n'en constitue pas moins sous forme déguisée, chez nombre d'écrivains ou de penseurs, la motivation inconsciente de leur activité créatrice. L'invention de la psychanalyse elle-même est quelque peu redevable au défi qui sans doute porta Freud, meurtri par l'antisémitisme de son temps, à répondre à et de l'humiliation du Juif dont il était le fils. ${ }^{21}$

En citant ces vers, Freud veut souligner une modalité de la transmission psychique mais aussi que la réception de l'héritage n'est pas passive, elle requiert l'engagement actif du sujet pour se l'approprier. René Kaës voit dans cet aphorisme la division du sujet de l'héritage comme celle du sujet de l'inconscient. Le sujet est divisé, comme Freud l'affirme dans Pour introduire le narcissisme, entre la double nécessité d'être "à lui-même sa propre fin et [le] maillon d'une chaîne à laquelle il est assujetti contre sa volonté ou du moins sans l'intervention de celle-ci ${ }^{22}$ " mais qu'il doit servir et sur le bénéfice de laquelle il peut compter. Il fait remarquer que cette double division, Freud la formule de façon très nette après Totem et tabou lorsqu'il envisage la question du narcissisme. Il oppose alors le statut narcissique du sujet à l'intersubjectivité, les liant ensemble et les articulant tous deux au point où le narcissisme de l'infans s'étaye sur celui de la génération précédente, la génération des parents qui lui transmettront les rêves qu'ils n'auront pas pu accomplir avec le souhait et la ferme conviction qu'il les accomplira. L'amour des parents, si touchant et si enfantin, n'est rien d'autre, écrira Freud, que leur narcissisme qui vient de renaitre.

Freud introduit ensuite une curieuse hypothèse - ce sera sa deuxième présupposition qui ne figurera plus dans aucune de ses œuvres: il ne la reprendra ni ne la discutera jamais plus. Il s'agit de cet «appareil que chaque homme possède dans son activité mentale inconsciente et qui lui permet d'interpréter les réactions d'autrui, c'est-à-dire d'annihiler de nouveau les déformations qu'autrui a imprimées à l'expression de ses sentiments ${ }^{23}$. " C'est un appareil à interpréter dont la caractéristique essentielle est sa fonction transformatrice. Il constitue et produit du sens. C'est l'appareil inconscient de la transmission. Par la compréhension inconsciente qu'il permet, il joue un rôle crucial dans la transmission de l'héritage affectif aux générations ultérieures, héritage issu de "toutes les coutumes, cérémonies et prescriptions que le rapport primitif au père originaire avait laissées derrière lui ${ }^{24}$. " 
L'héritage traumatique est bien ce qui met en échec cet appareil ne lui permettant pas de métaboliser l'objet transmis qui peut alors devenir persécuteur. Cette deuxième hypothèse soulève des questions qui, comme le souligne René Kaës, attendent des réponses que Freud n'a jamais élaborées; principalement celles qui concernent la constitution, le fonctionnement de cet appareil ainsi que sa modalité de transmission, à savoir la modification éventuelle de ce qui est transmis. Cependant l'appareil à penser dont parle Bion pourrait certainement nous aider à répondre à ces questions.

\section{Conclusion}

Que retenir de cette analyse? L'effondrement de l'interdit du meurtre désigne tout homme comme impunément tuable et crée une menace de meurtre qui abolit la distinction entre réalité psychique et la réalité matérielle. Cette menace de meurtre est génératrice d'une situation traumatique et se traduit par un état de détresse caractérisé par un afflux d'excitations qui met le pare-excitations hors jeu. Ce défaut du pareexcitations rend la transmission, traumatique, si l'on considère qu'il équivaut à un échec de la symbolisation. L'héritage à transmettre devient alors héritage traumatique où prédominent des éléments bruts, étranges, des objets irreprésentables dont l'origine est à chercher dans des vécus non élaborés ou en attente d'élaboration, éléments en souffrance qui peuvent traverser les générations et les espaces psychiques, devenir incompréhensibles ou indéchiffrables, jusqu'à parvenir à des héritiers dont les psychismes pourront éventuellement non seulement les contenir mais se les approprier en les transformant. Nous pouvons dire avec Albert Ciccone que le traumatisme se transmet sans se représenter ou se transmet pour ne pas être représenté2 ${ }^{25}$.

\section{NOTES}

1. La résistance de l'humain, sous la direction de Nathalie Zaltzman, PUF, Col. Petite bibliothèque de psychanalyse, 1999, p. 1.

2. Ibid, p. 2.

3. L'Homme Moïse et la religion monothéiste, Gallimard, 1986, p.131.

4. Adorno Th. W. (2003), Modèles critiques, Paris, Payot, 235-236.

5. Euvres, Tome XIII, p.151

6. Considérations, p.30.

7. La résistance de l'humain, PUF, Petite bibliothèque de psychanalyse, 1999, p.18.

8. Altounian Janine (2000), La survivance, traduire le trauma collectif, Inconscient et Culture, Dunod, p.25.

9. Ibid, p.19.

10. Levi Primo (1989), Les naufragés et les rescapés, Quarante ans après Auschwitz, Paris,Arcades, Gallimard, pp. 83-84

11. Freud Sigmund (1956 [1969], La naissance de la psychanalyse, Paris, Bibliothèque de psychanalyse, PUF, p191.

12. Balint Michaël (1977), Le défaut fondamental, Paris, Petite Bibliothèque Payot, p.207. 
13. Ibid, p.503

14. Forges Jean-François (2004), Éduquer contre Auschwitz, Histoire et mémoire, Agora, ESF éditeur, p.233

15. Pour Freud, il est à l'origine de la religion comme il le déclare dans une lettre à Jung du 2 janvier 1910 où il écrit: "La raison dernière du besoin de religion m'a frappé comme étant le désemparement [l'état de détresse] infantile, tellement plus grand chez l'homme que chez les animaux. À partir de ce moment il ne peut se représenter le monde sans parents, et s'octroie un Dieu juste et une nature bonne. Freud S. Jung C.G. (1992), Correspondance (1906-1914), Gallimard, p. 372

16. Decherf, Gérard avec Laurence Knéra et Élisabeth Darchis (2003), Souffrances dans la famille, Thérapie familiale psychanalytique d'aujourd'hui, Éditions In Press, p.66

17. Eiguer, Alberto (1997), Le générationnel, Collection Inconscient et Culture, Dunod, p.111

18. Op. cité, p. 4-5

19. Kaës René et al. (1989), Violence d'État et psychanalyse, Paris, Inconscient et Culture, Dunod, p. 173

20. Ibid, p.314

21. Altounian J. (2000), La survivance, Traduire le trauma collectif, Paris, Inconscient et Culture, Dunod, p136-137

22. Freud S. (1914 [1969]), Pour introduire le narcissisme in La vie sexuelle, Paris, PUF, p.85

23. Freud S. (1913 [1993]), Totem et tabou, Paris, Gallimard, Connaissance de l'inconscient, p.315

24. Totem et tabou, p. 315

25. Ciccone Albert (1999), La transmission psychique inconsciente, Dunod, p. 95.

\section{AUTEUR}

\section{DAVID BENHAIM}

Psychanalyste 